\title{
USE OF RESPONSE ENVELOPES FOR SEISMIC MARGIN ASSESSMENT OF REINFORCED CONCRETE WALLS AND SLABS
}

\author{
Alberto FRAU ${ }^{1}$, Nicolas ILE ${ }^{2}$ \\ ${ }^{1}$ Den-Service d'étude mécanique et thermique (SEMT), CEA, Université Paris-Saclay, F-91191, Gif-sur-Yvette. \\ alberto.frau@cea.fr \\ ${ }^{2}$ Den-Service d'étude mécanique et thermique (SEMT), CEA, Université Paris-Saclay, F-91191, Gif-sur-Yvette. \\ nicolas.ile@cea.fr
}

\begin{abstract}
Seismic safety evaluations of existing nuclear facilities are usually based on the assumption of structural linearity. For the design basis earthquake (DBE), it is reasonable to apply a conventional evaluation of the seismic safety of building structures and carry out a linear elastic analysis to assess the load effects on structural elements. Estimating the seismic capacity of a structural element requires an estimation of the critical combination of responses acting in this structural element and compare this combination with the capacity of the element. By exploiting the response-spectrum-based procedure for predicting the response envelopes in linear structures formulated by Menun and Der Kiureghian (2000) [13], algorithms are developed for the seismic margin assessment of reinforced concrete shell finite elements. These algorithms facilitate the comparison of the response-spectrum-based envelopes to prescribed capacity surfaces for the purpose of assessing the safety margin of this kind of structures. The practical application of elliptical response envelopes in case of shell finite elements is based on the use of layer models such as those developed by Marti (1990) [11], which transfer the generalized stress field to three layers under the assumption that the two outer layers carry membrane forces and the internal layer carries only the out-of-plane shears. The utility of the assessment approach is discussed with reference to a case study of a 3D structure made of reinforced concrete walls.
\end{abstract}

\section{INTRODUCTION}

Nuclear safety standards as well as building codes appoint the response spectrum method as the preferred technique for structural analysis and design, see for example EPRI (1994) [6] and Eurocode 8 [4]. For each mode, the individual peak value of the response quantity of interest is computed, using a prescribed set of response spectra. These maxima are then combined using a suitable combination rule. In general a common approach is CQC (Complete Quadratic Combination) proposed by Der Kiureghian (1979) [8]. In order to combine the effects of the three directions motion two others rules are used: SRSS (Square Root of the Sum of the Squares) or $40 \%$ rules. Using the classical spectral method the main idea is to determine the maximum value of each single quantities and to compare this with the single demand quantities. If the hypothesis of these maxima occur simultaneously, the response vector is represented by a rectangular envelope. This assumption may be overly conservative for many assessment or design situations. Menun and Der Kiureghian (2000) ([13], [14]) proposed an improved envelope based on random vibration theory that takes into account the correlation between the response components. In this case the interacting response components belong to an elliptical response envelope which is inscribed within the rectangular envelope specified by the individual peak values. Menun and Der Kiureghian (2000) [13] and Menun (2004) [15] have developed two efficient algorithms for the purpose of either assessing the available safety margin of an existing design of a structural element or determining the required value of a design parameter. Menun (2004) [15] show that using the elliptical envelope, higher safety margins could be calculated compared to the classical rectangular envelope.

In this paper, we define a robust algorithm that can be used to calculate the safety margins for slabs and walls. We compare the response-spectrum-envelope with the capacity surface of the element defined for the threelayer model of (Marti, 1990 [11]). In this approach the generalized forces are transferred to three layers: 2 outer layers carry the membrane forces and an internal layer carries only the out-of-plane efforts. 


\section{ELLIPTICAL RESPONSE ENVELOPE FOR SEISMIC ASSESSMENT OF REINFORCED CONCRETE SHELL ELEMENTS}

Menun and Der Kiureghian (2000) ([13], [14]) developed a procedure for predicting the envelope that bounds the response vector for a structure under the hypothesis of linear behaviour for an earthquake excitation which is defined by two horizontal components and one vertical component. The upper bound of the interaction responses is represented by an elliptical envelope. Using several time-history motions, where the principal components are well-known, Menun (2004) [15] show that the elliptical envelope bounds the response vector in an average sense. For the general case, in which the orientation of the principal components of ground motion is unknown, the same authors defined a further surface, denominated supreme envelope.

A general point (an n-vector of responses) on the elliptical envelope (Figure 1) is defined using the equation (1):

$$
x_{\alpha}=x_{O \alpha}+\frac{X_{\alpha} \alpha}{\sqrt{\alpha^{T} X_{\alpha} \alpha}}
$$

Where $\boldsymbol{\alpha}$ is the unit normal n-vector of the hyperplane $P_{\alpha} ; \boldsymbol{x}_{O \alpha}$ is the time-invariant n-vector of responses (static response) and $\boldsymbol{X}_{\boldsymbol{\alpha}}$ is the a symmetric $n \times n$ "response matrix". The diagonal elements of the "response matrix" are the squared peak values of the individual response components, while the off diagonal terms are related to the covariance of the response components. The expressions of each terms are defined in the equations (2) and (3):

$$
\begin{aligned}
& X_{r}^{2}=\sum_{k=1}^{3} \sum_{i=1}^{n} \sum_{j=1}^{n}\left(q_{r}^{T} \phi_{i} \gamma_{k i}\right)\left(q_{r}^{T} \phi_{j} \gamma_{k j}\right) \rho_{i j} S_{k i} S_{k j} \\
& X_{r s}=\sum_{k=1}^{3} \sum_{i=1}^{n} \sum_{j=1}^{n}\left(q_{r}^{T} \phi_{i} \gamma_{k i}\right)\left(q_{s}^{T} \phi_{j} \gamma_{k j}\right) \rho_{i j} S_{k i} S_{k j}
\end{aligned}
$$

Where $q_{r}$ and $q_{s}$ are the functions of the stiffness and undeformed geometry of the structure, $\phi_{i}$ and $\phi_{j}$ are the $i-t h$ and $j-t h$ mode shape, $\gamma_{k i}$ and $\gamma_{k j}$ is the modal participation factor corresponding to modes $i$ and $j$ for the $k-t h$ principal component of ground motion, $\rho_{i j}$ denotes the correlation coefficient between the $i-t h$ and $j-t h$ modal responses and $S_{k i}$ and $S_{k j}$ represent the relative displacement response spectrum ordinates corresponding to modes $i$ and $j$ for the $k-t h$ principal component of ground motion.

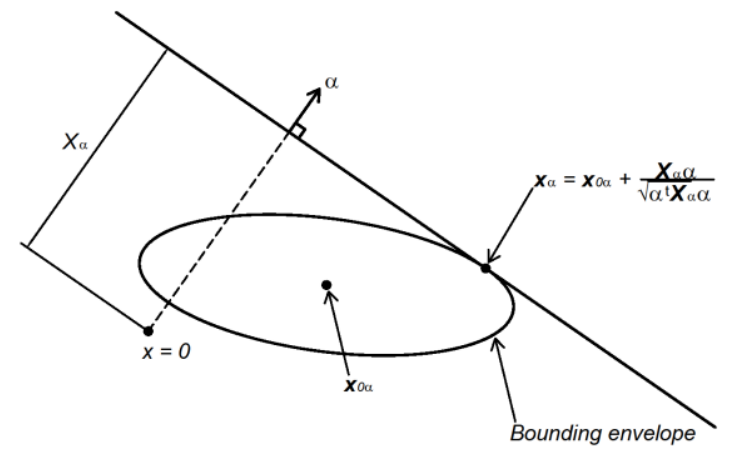

Figure 1. Geometrical characterization of the response-spectrum-based envelope.

In the case where the $r$ and $s$ components are uncorrelated the terms $X_{r s}$ are equal to zero. Besides, if the response is perfectly correlated the norm of (3) is equal to $X_{r} X_{s}$.

For shell elements the dimension of the response vector is equal to 8. In order to define the "response matrix" the equations (2) and (3) are valid. Besides, if we want to calculate the safety margins, it's necessary to construct a capacity or a limit domain in the 8-dimensional space. This task is not straightforward, firstly because of the mathematical complexity of the problem and secondly, because of the lack of experimental data.

Several theoretical models for the design and analysis of reinforced concrete plates and shells exist in the literature (Capra and Maury (1978) [2]; Marti (1990) [11]; Laurenço and Figueiras (1995) [10]; Palacio (2007) [16]; Jaeger (2014) [9]). Basically, most of them are sandwich type models of two or three layers. In this framework we use the three-layer model of Marti (Marti (1990) [11]; Palacio (2007) [16]). The shell element is split in three layers: two external layers carry membrane forces due to the six externally applied components 
per unit of length $\left(n_{x}, n_{y}, n_{x y}, m_{x}, m_{y}, m_{x y}\right.$ - Figure 2) while the intermediate layer has the task of carrying the transverse (out-of-plane) shear forces $V_{x}$ and $V_{y}$. In order to define this layers, the thickness of the external layers $c$ is supposed equal to 2 times of the concrete cover and the middle plane is located in the reinforcement mesh barycentre. If $h$ is the thickness of the shell element the lever arm $d$ is equal to $h-c$. The resultant membrane forces per unit length for the external layers (top - Eqs (4) - (6) - and bottom - Eqs (7) - (9)) are:

$$
\begin{aligned}
N_{x} & =\frac{n_{x}}{2}+\frac{m_{x}}{d}+\frac{V_{x}^{2}}{2 V_{0} \tan \left(\theta_{v}\right)} \\
N_{y} & =\frac{n_{y}}{2}+\frac{m_{y}}{d}+\frac{V_{y}^{2}}{2 V_{0} \tan \left(\theta_{v}\right)} \\
N_{x y} & =\frac{n_{x y}}{2}+\frac{m_{x y}}{d}+\frac{V_{x} V_{y}}{2 V_{0} \tan \left(\theta_{v}\right)} \\
N_{x} & =\frac{n_{x}}{2}-\frac{m_{x}}{d}+\frac{V_{x}^{2}}{2 V_{0} \tan \left(\theta_{v}\right)} \\
N_{y} & =\frac{n_{y}}{2}-\frac{m_{y}}{d}+\frac{V_{y}^{2}}{2 V_{0} \tan \left(\theta_{v}\right)} \\
N_{x y} & =\frac{n_{x y}}{2}-\frac{m_{x y}}{d}+\frac{V_{x} V_{y}}{2 V_{0} \tan \left(\theta_{v}\right)}
\end{aligned}
$$

where $V_{0}=\sqrt{V_{x}^{2}+V_{y}^{2}}$.

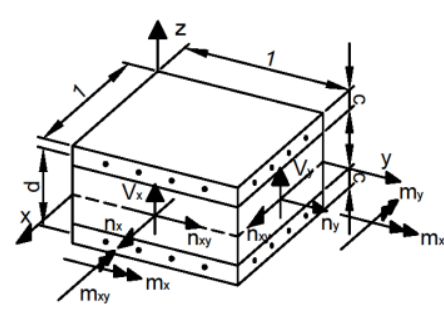

(a)

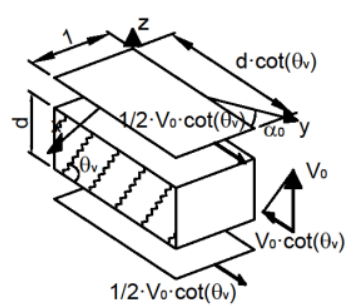

(c)

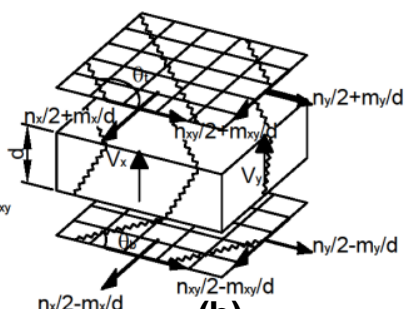

(b)

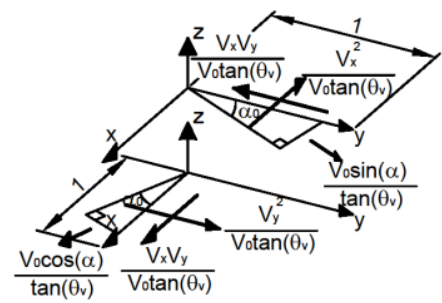

(d)

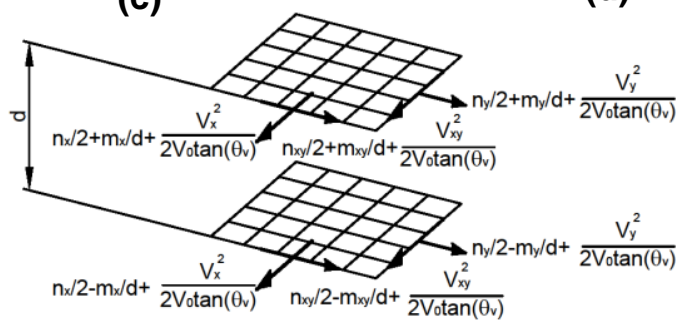

(e)

Figure 2. Three-layer sandwich model of Marti (1990) [11]: a) definition, b) outer and inner layers, c) diagonal compression field in the cracked inner layer, d) membrane forces equilibrating $V_{0} \cot \left(\theta_{v}\right)$, e) forces acting in the outer layers.

In the inner layer the shear forces $V_{x}$ and $V_{y}$ are carried with the help of the truss mechanism. The angle $\theta_{v}$ is the inclination of the diagonal concrete compressive struts. Marti (1990) [11] suggest that this value must be between $25^{\circ}$ and $45^{\circ}$. In this paper, we consider $\theta_{v}=35^{\circ}$. Using the three-layer model, we are able to split 
the problem in two parts (external and internal layers). The dimension of the response vector pass from 8 to 3 for the external layers and 2 for the inner layer. Thus, the "response matrix" for the external (10) and the internal (11) layers is:

$$
\boldsymbol{X}_{\boldsymbol{\alpha}}=\left[\begin{array}{ccc}
N_{x}^{2} & N_{x, y} & N_{x, x y} \\
N_{x, y} & N_{y}^{2} & N_{y, x y} \\
N_{x, x y} & N_{y, x y} & N_{x y}^{2}
\end{array}\right] \quad \text { (10) } \quad \boldsymbol{X}_{\boldsymbol{\alpha}}=\left[\begin{array}{cc}
V_{x}^{2} & V_{x, y} \\
V_{x, y} & V_{y}^{2}
\end{array}\right]
$$

\section{YIELD CRITERIA FOR REINFORCED CONCRETE MEMBRANE ELEMENTS}

In order to calculate the safety seismic margins, it is necessary to compare the response envelope with the capacity domain issues on the ultimate or yield conditions. Using the Marti's model, it is necessary to define two different capacity domains: the first one corresponds to the membrane limit state of the two external layers and the second one corresponds to the transverse shear limit state of the inner layer.

\section{Capacity domain for the membrane limit state (external layers)}

Several methodologies are proposed by some authors (Palacio (2007) [16] and Meyboom (2002) [12]) for the $\mathrm{RC}$ membrane members in plane stress. In this section we present the methodology used in this article.

Starting a classical configuration for a RC membrane elements with orthogonally steel bars in $\mathrm{x}-$ and $\mathrm{y}$ direction (Figure 3), it is possible to decompose the applied stress $\left(\sigma_{x}, \sigma_{y}\right.$ and $\left.\sigma_{x y}\right)$ in two parts: one for the concrete $\left(n_{c x}, n_{c y}\right.$ and $\left.n_{c x y}\right)$ and the second one for the steel $\left(n_{s x}\right.$ and $\left.n_{s y}\right)$.

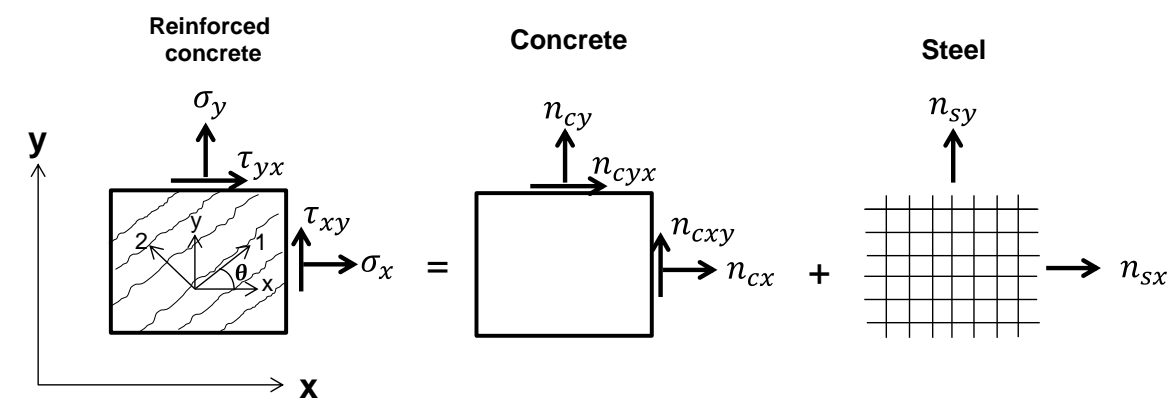

Figure 3. Reinforced concrete membrane element subjected to in-plane stress.

For the concrete part, after cracking, where the concrete is separated in struts orientated by an angle $\theta$, the stress tensor $\left[n_{c x}, n_{c y}, n_{c x y}\right]$ can be expressed in terms of principal stress:

$$
\begin{gathered}
n_{c x}=n_{c 2} \cdot \sin ^{2}(\theta)+n_{c 1} \cdot \cos ^{2}(\theta) \\
n_{c y}=n_{c 2} \cdot \cos ^{2}(\theta)+n_{c 1} \cdot \sin ^{2}(\theta) \\
n_{c x y}=\left(n_{c 1}-n_{c 2}\right) \cdot \sin (\theta) \cdot \cos (\theta)
\end{gathered}
$$

Combining the equations (12) - (14) it is possible to rewrite the problem in another form:

$$
\begin{aligned}
& \left(n_{c x}-n_{c 1}\right) \cdot\left(n_{c y}-n_{c 1}\right)=n_{c x y}^{2} \\
& \left(n_{c x}-n_{c 2}\right) \cdot\left(n_{c y}-n_{c 2}\right)=n_{c x y}^{2}
\end{aligned}
$$

According to the typical stress-strain behaviour of the concrete in uniaxial compression and tension (Figure $4 \mathrm{a}$, we consider that the failure occurs when the terms $n_{c 1}$ and $n_{c 2}$ reach the compressive stress $-f_{c}$. No tensile stress is allowed. Thus, the equations (15) and (16) define two regimes:

$$
\begin{gathered}
\Phi_{c 1}=n_{c x} \cdot n_{c y}-n_{c x y}^{2} \geq 0 \text { for } n_{c x} \leq 0, n_{c y} \leq 0 \text { and }\left(n_{c x}+f_{c}\right)+n_{c y}>0 \\
\Phi_{c 2}=\left(n_{c x}+f_{c}\right) \cdot\left(n_{c y}+f_{c}\right)-n_{c x y}^{2} \geq 0 \text { for } n_{c x} \geq-f_{c}, n_{c y} \geq-f_{c} \text { and }\left(n_{c x}+f_{c}\right)+n_{c y} \leq 0
\end{gathered}
$$

The yield surface defined by the equations (17) and (18) is presented in Figure 4b. According the Eurocode 2 [5] the term $f_{c}$ is given by:

$$
f_{c}=v f_{c d} \quad f_{c d}=\alpha_{c c} f_{c k} / \gamma_{c} \quad v=0.6\left(1-f_{c k} / 250\right)
$$


Where $v$ is the reduction factor of the strength of cracked concrete due to shear; $f_{c d}$ is the design concrete compressive strength, expressed in $\mathrm{MPa} ; f_{c k}$ is the characteristic concrete compressive strength, expressed in $\mathrm{MPa} ; \alpha_{c c}$ is the coefficient taking account of the long term effects on the compressive strength (the recommended value is 1$)$ and $\gamma_{c}$ is the partial safety factor for concrete (1.5 for persistent loading and transient loading and 1.2 for accidental loading).
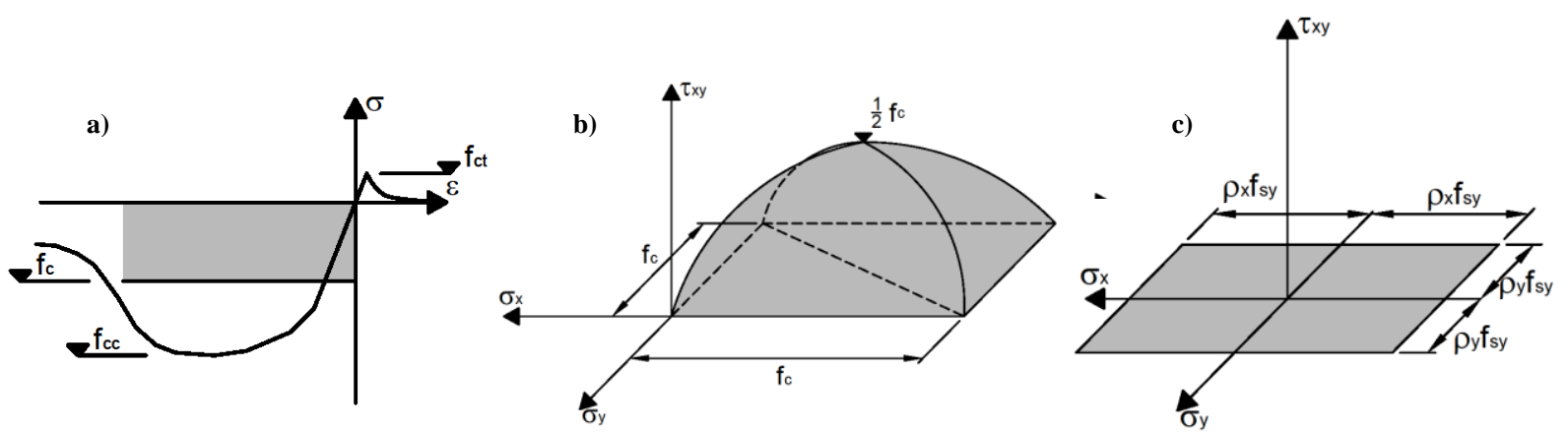

Figure 4. (a) stress-strain curve for uniaxially loaded concrete; (b) yield criterion for plain concrete without tension; (c) yield criterion for reinforcement.

For the steel part $\left(\left[n_{s x}, n_{s y}, 0\right]\right)$ the yield criterion is fixed by the classical rigid-perfectly plastic behavior (Figure 4c). Each terms $n_{s i}$ are bounded between the values $-\rho_{i} f_{s y}$ and $\rho_{i} f_{s y}$ (where the index $i$ is equal to $x$ and $y$ ). According the Eurocode 2 [5] the $f_{s y}$ terms are obtained from the characteristic yield strength value $\left(f_{s k}\right)$ divided by the safety factor $\gamma_{s}$ (1.15 for persistent and transient loading and 1.0 for accidental loading) Combining the two domains for concrete and steel, the following yield criteria are obtained, characterizing the seven yield regimes (Figure 5):

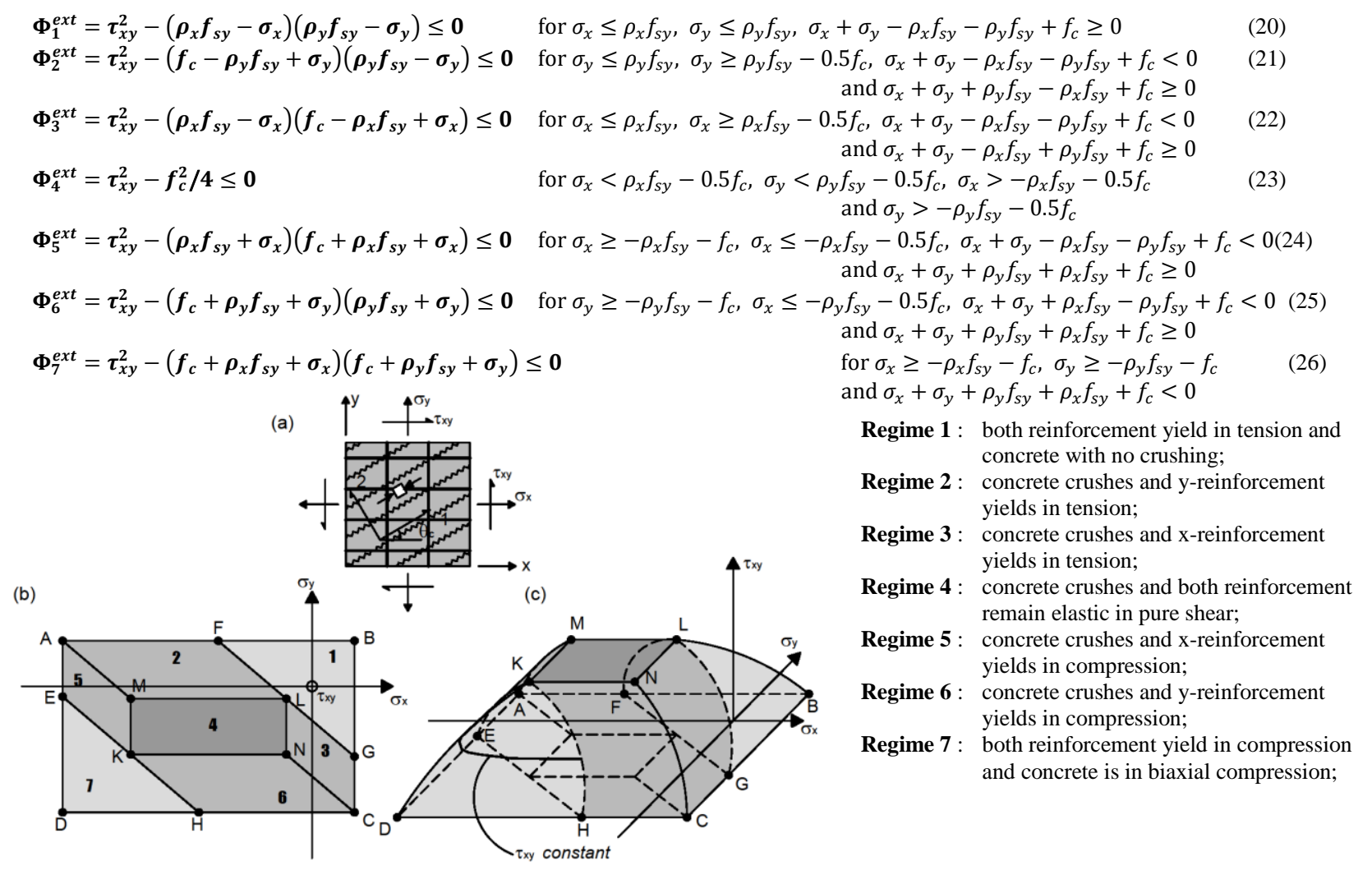

Figure 5. Reinforced concrete membrane element - a) element subjected to in-plane stress; b) identification of yield regimes; c) yield surface of reinforced concrete. 


\section{Capacity domain for the transverse shear limit state (inner layer)}

For the inner layer, the yield domain is represented by a limitation of the shear stress given by the equation (27):

$$
\tau_{\text {calc }}=\frac{\sqrt{V_{x}^{2}+V_{y}^{2}}}{d}=\frac{V_{0}}{d} \leq \tau_{R d, c}
$$

Where $d$ is the lever arm and $\tau_{R d, c}$ is the design shear stress resistance. In the regions with transverse shear reinforcement, this one is equal to :

$$
\begin{aligned}
& \tau_{R d, c}=\min \left\{\tau_{R d, s} ; \tau_{R d, \max }\right\} \text { where } \tau_{R d, s}=\rho_{z} \cdot f_{y w d} \cdot \cot \theta \\
& \tau_{R d, \max }=\alpha_{c w} \cdot v_{1} \cdot f_{c d} /(\cot \theta+\tan \theta)
\end{aligned}
$$

Where $\rho_{z}$ is the transverse reinforcement ratio; $f_{y w d}=f_{y k} / \gamma_{s}$ is the design yield strength of the shear reinforcement; $\alpha_{c w}$ is a coefficient that takes into account the effect of normal stresses on the shear strength (equal to 1 for non-prestressed structures); $v$ is a coefficient that takes into account the increase of fragility and the reduction of shear transfer by aggregate interlock with the increase of the compressive concrete strength (6 for $f_{c k} \leq 60 \mathrm{MPA}$ and $0.9-f_{c k} / 200>0.5$ for $\left.f_{c k}>60 \mathrm{MPa}\right)$ and $\theta$ is the angle between the inclined struts $(1 \leq \cot (\theta) \leq 2.5)$. Without transverse shear reinforcement $\tau_{R d, c}$ is equal to $0.35 / \gamma_{c} \cdot f_{c k}\left(f_{c k}\right.$ in $\left.\mathrm{MPa}\right)$. The capacity domain is given by the equation (29):

$$
\Phi^{i n n}=\tau_{c a l c}-\tau_{R d, c} \leq 0
$$

\section{MARGINS ASSESSMENT ALGORITHMS}

In order to verify whether the response envelope bounding the response vector is completely contained within the capacity surface and to obtain a valid measure of the seismic safety margin an iterative procedure is needed. To find the seismic safety margin, the response matrix (10) et (11) are amplified by a scalar $\lambda$ (safety margin) where the envelope (generated by ultimate response matrix $\widehat{\boldsymbol{X}}_{\boldsymbol{\alpha}}^{\boldsymbol{\sigma}}$ ) must be tangent to the yield surface at the point of the ultimate stress state $\widehat{\boldsymbol{x}}_{\boldsymbol{\alpha}}^{\sigma}$.

$$
\widehat{x}_{\boldsymbol{\alpha}}^{\sigma}=x_{\boldsymbol{0} \alpha}^{\sigma}+\frac{\widehat{X}_{\alpha}^{\sigma} \boldsymbol{\alpha}}{\sqrt{\boldsymbol{\alpha}^{T} \widehat{X}_{\alpha}^{\sigma} \boldsymbol{\alpha}}} \quad \text { where } \widehat{X}_{\boldsymbol{\alpha}}^{\sigma}=\lambda^{2} \cdot \boldsymbol{X}_{\boldsymbol{\alpha}}^{\sigma}
$$

The following additional two conditions have to be satisfied:

$$
\begin{gathered}
\Phi\left(\widehat{\boldsymbol{x}}_{\boldsymbol{\alpha}}^{\boldsymbol{\sigma}}\right)=0 \\
\left|\frac{\partial \Phi}{\partial \sigma}\right|_{\boldsymbol{\sigma}=\widehat{\boldsymbol{x}}_{\boldsymbol{\alpha}}^{\sigma}}=\boldsymbol{\alpha}
\end{gathered}
$$

The condition (31) ensures that the envelope is in contact with the yield surface, whereas equation (32) guarantees the tangency condition. The safety margin $\lambda$ must be the minimum that satisfies conditions (30)(32).

Note that the terms $\boldsymbol{x}_{\mathbf{0} \boldsymbol{\alpha}}^{\boldsymbol{\sigma}}, \widehat{\boldsymbol{x}}_{\boldsymbol{\alpha}}^{\boldsymbol{\sigma}}, \widehat{\boldsymbol{X}}_{\boldsymbol{\alpha}}^{\boldsymbol{\sigma}}$ and $\boldsymbol{X}_{\boldsymbol{\alpha}}^{\boldsymbol{\sigma}}$ are the static, the ultimate seismic response, the ultimate and current response matrix. Thus, the terms $\widehat{x}_{\mathbf{\alpha} \boldsymbol{\alpha}}^{\sigma}$ and $\boldsymbol{X}_{\boldsymbol{\alpha}}^{\boldsymbol{\sigma}}$ for the two external layers the inner layer are defined as follow:

$$
x_{0 \alpha}^{\sigma}=x_{0 \alpha} / c X_{\alpha}^{\sigma}=X_{\alpha} / c^{2} \text { (external layer) } x_{0 \alpha}^{\sigma}=x_{0 \alpha} /(d-c) X_{\alpha}^{\sigma}=X_{\alpha} /(d-c)^{2} \text { (inner layer) }
$$

The problem defined by equations (45) - (48) is a nonlinear optimization problem and its solution can be obtained by means of the following Newton-Raphson algorithm:

1. Define several initial direction $\boldsymbol{\alpha}_{0}^{i}$ where $i=1 \ldots n_{\alpha}$, where $n_{\alpha}=6$ for the three-dimensional space and $n_{\alpha}=4$ in the two-dimensional space;

2. In order to find the scalar $\lambda^{i}$, loop for $i=1 \ldots n_{\alpha}$;

Using the equation (30), for the scalar $\lambda_{0}^{i}=1.0$, we obtain the quantities $\boldsymbol{x}_{\boldsymbol{\alpha}, 0}^{\boldsymbol{\sigma}, i}$. With equation $\boldsymbol{x}_{\boldsymbol{\alpha}, 0}^{\boldsymbol{\sigma}, i}=$ $R_{0}^{i} B_{0}^{i}+\boldsymbol{x}_{\mathbf{0} \boldsymbol{\alpha}}^{\boldsymbol{\sigma}}$, we define the following quantities: 


$$
\boldsymbol{B}_{0}^{i}=\left(\boldsymbol{x}_{\boldsymbol{\alpha}, 0}^{\boldsymbol{\sigma}, i}-\boldsymbol{x}_{\mathbf{0} \boldsymbol{\alpha}}^{\boldsymbol{\sigma}}\right) / S_{0}^{i} S_{0}^{i}=\left|\boldsymbol{x}_{\boldsymbol{\alpha}, 0}^{\boldsymbol{\sigma}, i}-\boldsymbol{x}_{\mathbf{0} \boldsymbol{\alpha}}^{\boldsymbol{\sigma}}\right| R_{0}^{i}=S_{0}^{i}
$$

2A. In order to guarantee the condition (32), loop for $j=0 \ldots n_{j}$ :

if $j=0$

$\boldsymbol{\alpha}_{j}^{i}=\boldsymbol{\alpha}_{0}^{i} \quad \boldsymbol{x}_{\boldsymbol{\alpha}, j}^{\boldsymbol{\sigma}, i}=\boldsymbol{x}_{\boldsymbol{\alpha}, 0}^{\boldsymbol{\sigma}, i} \boldsymbol{B}_{j}^{i}=\boldsymbol{B}_{0}^{i} S_{j}^{i}=S_{0}^{i}$

Otherwise:

Using the vector $\boldsymbol{\alpha}_{j}^{i}$, the quantities $\boldsymbol{x}_{\boldsymbol{\alpha}, j}^{\boldsymbol{\sigma}, i}, \boldsymbol{B}_{j}^{i}, S_{j}^{i}$ and $R_{j}^{i}$ are computed using the equations (34) and (30).

2B. Condition (31) is satisfied with an internal loop for $k=0 \ldots n_{k}$;

For $k=0$ :

$R_{j, k}^{i}=R_{j, 0}^{i}=R_{j}^{i} \quad$ and $\quad x_{\alpha, j, k}^{\boldsymbol{\sigma}, i}=\boldsymbol{x}_{\boldsymbol{\alpha}, j, 0}^{\boldsymbol{\sigma}, i}=\boldsymbol{x}_{\boldsymbol{\alpha}, j}^{\boldsymbol{\sigma}, i}$

If $\Phi\left(R_{j, k}^{i} \boldsymbol{B}_{j}^{i}+\boldsymbol{x}_{\mathbf{0} \alpha}^{\sigma}\right)<$ tol (small tolerance), quit the loop $k$ and the new value of $\lambda_{j}^{i}$ is:

$\lambda_{j}^{i}=R_{j, k}^{i} / S_{j}^{i}$

Otherwise the condition (31) is satisfied with the classical Netwon-Rapson technique:

$R_{j, k+1}^{i}=R_{j, k}^{i}-\Phi\left(R_{j, k}^{i} \boldsymbol{B}_{\boldsymbol{j}}^{\boldsymbol{i}}+\boldsymbol{x}_{0 \alpha}^{\sigma}\right) / D\left(R_{j, k}^{i} \boldsymbol{B}_{\boldsymbol{j}}^{\boldsymbol{i}}+\boldsymbol{x}_{0 \alpha}^{\sigma}\right)$

Where :

$D\left(R_{j, k}^{i} \boldsymbol{B}_{j}^{i}+\boldsymbol{x}_{0 \alpha}^{\sigma}\right)=\left[\Phi\left(\left(R_{j, k}^{i}+\Delta\right) \boldsymbol{B}_{j}^{i}+\boldsymbol{x}_{0 \alpha}^{\sigma}\right)-\Phi\left(\left(R_{j, k}^{i}-\Delta\right) \boldsymbol{B}_{j}^{i}+\boldsymbol{x}_{0 \alpha}^{\sigma}\right)\right] / 2 \Delta$ and $\Delta=S_{j}^{i} / 10^{4}(58)$

Go to $2 \mathrm{~B}$. In the equation (36) the terms $R_{j, k+1}^{i} \rightarrow R_{j, k}^{i}$;

When the condition (31) is satisfied, the condition of normality is verified. If $\left|\frac{\partial \Phi}{\partial \sigma}\right|_{\sigma=R_{j, k}^{i} B_{j}^{i}+x_{0 \alpha}^{\sigma}}$ verifies the following condition:

$\left|\frac{\partial \Phi}{\partial \sigma}\right|_{\sigma=R_{j, k}^{i} B_{j}^{i}+x_{0 \alpha}^{\sigma}}-\boldsymbol{\alpha}_{j}^{i} \mid<t o l$

Quit the loop $j$ and $\lambda^{i}=\lambda_{j}^{i}$; otherwise, the new direction $\boldsymbol{\alpha}$ is choses as follow:

$\boldsymbol{\alpha}_{j+1}^{i}=\left.\frac{\partial \Phi}{\partial \sigma}\right|_{\sigma=R_{j, k}^{i} B_{j}^{i}+x_{0 \alpha}^{\sigma}}$

Go to the 2A. In the equation (30) and (30) $\boldsymbol{\alpha}_{j+1}^{i} \rightarrow \boldsymbol{\alpha}_{j}^{i}$

3. The seismic safety factor is equal to $\lambda=\min \lambda^{i}$;

The same algorithm is used for the two external layers and the inner layer. In the first case the yield surface $\Phi$ is defined by the equations (20) - (26) and the conditions (31) - (32) define a three-dimensional problem. For the inner layer, the problem becomes two-dimensional and the yield surface is defined by equation (29).

\section{APPLICATION: SEISMIC VERIFICATION OF A 3D STRUCTURE MADE OF RC}

The results obtained by applying the solution strategies presented in sections 2 to 5 to a 3 -storey asymmetric reinforced concrete specimen are presented here. The specimen is a $1 / 4$ scaled model representing a simplified part of a nuclear electrical building. It has been constructed to reproduce in the best possible way the geometrical and dynamic characteristics of a part of a real nuclear building. The specimen was subjected to increasing bi-directional seismic signals. The data provided by the experimental program have been used for the international benchmark SMART2013 in order to validate nonlinear numerical models, to set a basis for a benchmark exercise [17], and to quantify the seismic margin with respect to the design level.

The specimen was designed according to the current French design rules (Gupta O, Lacoste AC., (2006) [7]). Seismic excitation in the $\mathrm{X}$ and $\mathrm{Y}$ directions is represented by the same elastic response spectrum having a PGA equal to $0.2 \mathrm{~g}$. In the vertical direction, the design spectrum is equal to the horizontal spectrum multiplied by $2 / 3$ for all frequencies. The design spectra are shown in Figure 6 a. 
a)

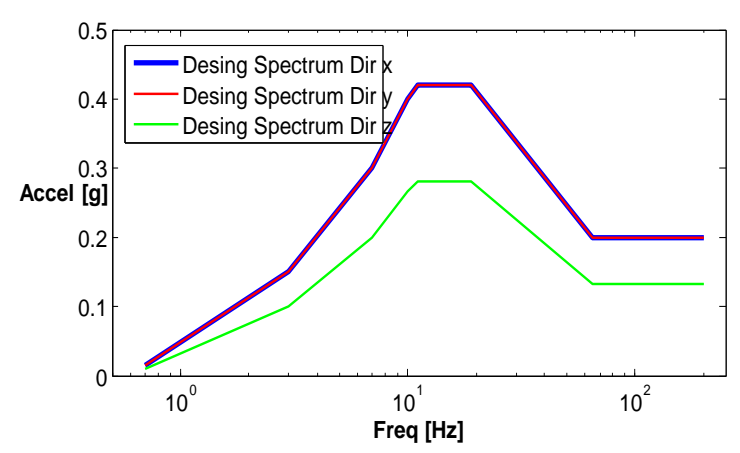

b)

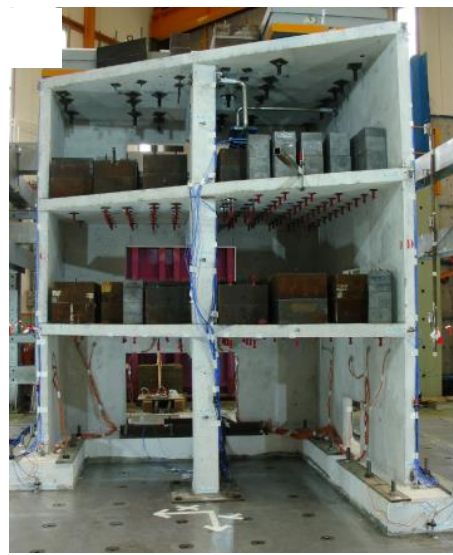

c)

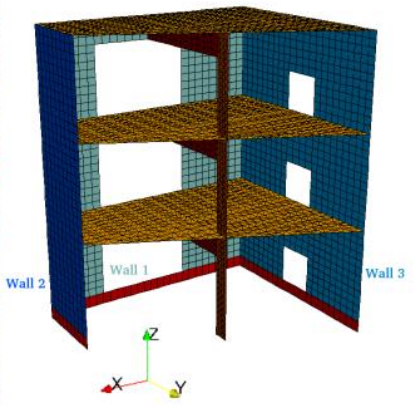

Figure 6. a) Design response spectra - b) Picture of the specimen; c) Mesh used for the FEM analysis.

The structure is a 3-storey reinforced concrete building with a strong asymmetry (Figure 6a). Figure 6b shows a picture of the specimen and the FEM mesh used for the analysis. In Figure 7 we show the main dimensions for the three walls that composed the structure with the distribution of the reinforcement ratio for the two external layers and the inner layer $\left(\rho_{h}\right.$ represents the horizontal reinforcement ratio, $\rho_{v}$ stands for the vertical reinforcement ratio and $\rho_{z}$ is the transverse reinforcement ratio). The mechanical properties of the concrete are: $f_{c k}=30 \mathrm{MPa}$ (characteristic strength), $v_{c o n}=0.2$ (Poisson coefficient) $E_{\text {con }}=32000 \mathrm{MPa}$ (Young's modulus). For the steel of the reinforcing bars, the characteristic strength is equal to $500 \mathrm{MPa}$. More details could be found in [17].

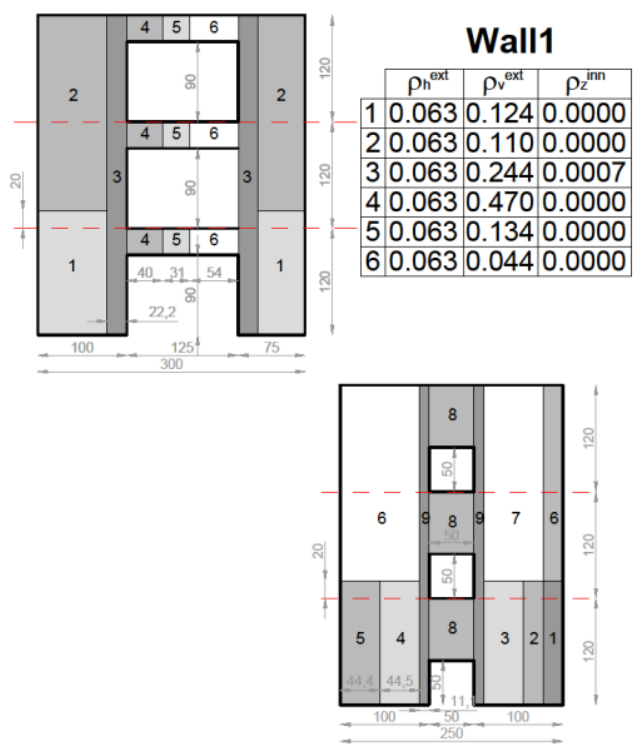

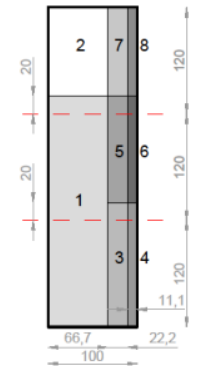

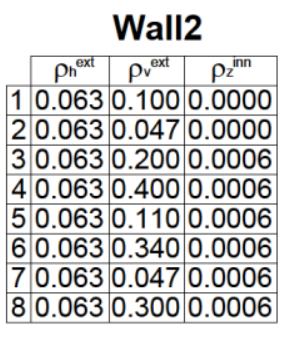

Wall3

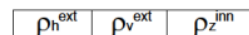

10.0630 .2400 .0006

20.0630 .1040 .0000

30.0630 .0600 .0000

\begin{tabular}{l|l|l|l|l|l|}
\hline 4 & 0.063 & 0.050 & 0.0000
\end{tabular}

50.0630 .1100 .0000

\begin{tabular}{l|l|l|l|l|}
6 & 0.063 & 0.158 & 0.0006
\end{tabular}

\begin{tabular}{llll|l}
7 & 0.063 & 0.045 & 0.0000
\end{tabular}

80.0630 .1000 .0000

$90.0630 .250 \quad 0.0007$

Figure 7. Arrangement and reinforcement ratios for Wall1, Wall2 and Wall3.

Using the Cast3M FEM Code [3], the analysis is carried out for the three walls using the elliptical and the rectangular response-spectrum-based envelopes. In this section we want to discuss about the intrinsic conservatism when the covariance terms are taken into accounts. In Figure 8 we compare the safety margins $\lambda$, for the external layer of the Wall 1, for each strategy. The two strategies give a similar distribution of the safety margin throughout the wall and, as expected, the minimum safety margins is located where a concentration of stress appears, for example for opening or the connections between the walls (Figure 8a and $8 \mathrm{~b})$. As expected, the lower $\lambda$ values are obtained when rectangular envelope strategy is employed. This aspect is also illustrated in Figure 8c, where the ratio $\lambda^{\text {elli }} / \lambda^{\text {para }}$ is always greater than 1 , the maximum value being 
about 1.30. For this example, it was observed that $\lambda^{\text {elli }} / \lambda^{\text {para }}$ values less than 1.10 were obtained for elements whose response components are generally strongly correlated (the ellipsoid being tangent to the parallelepiped in a point close to a corner).

(a)

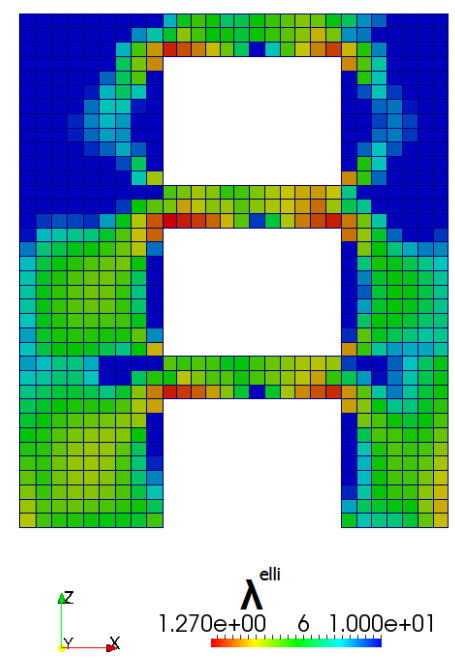

(b)
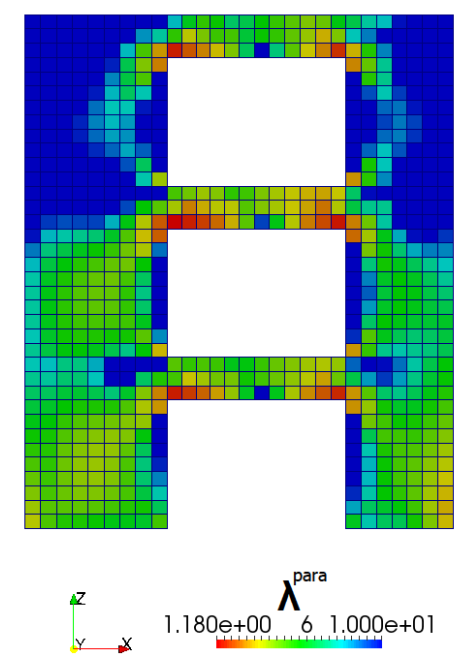

(c) Wall - Ratio $\lambda^{\text {elli }} / \lambda^{\text {para }}$
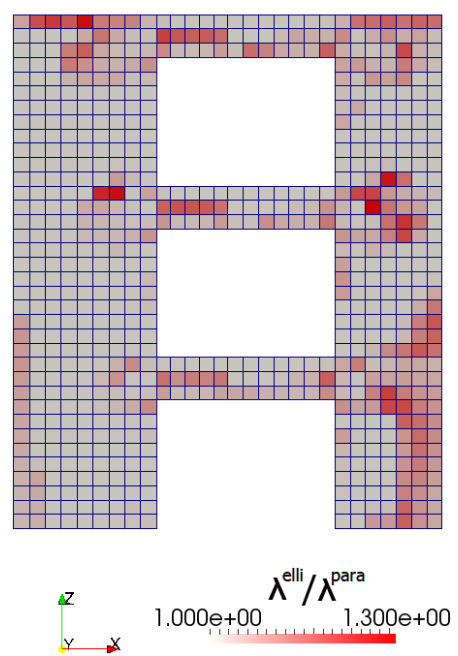

Figure 8. Comparison of the seismic safety margin $\lambda^{\text {elli }}$ (a) and $\lambda^{\text {para }}$ (b) for the external layer (top), gathered using the ellipsoid and parallelepiped envelopes respectively, and the ratio $\lambda^{e l l i} / \lambda^{\text {para }}$.

The higher values of $\lambda^{\text {elli }} / \lambda^{\text {para }}$ ratio were calculated for elements having lower correlation between response components. An example is shown in Figure 9, where ultimate parallelepiped and ultimate ellipsoid envelopes for the element 378 are compared, the seismic margin given by the elliptical envelope being about $16 \%$ higher than that given by the classical rectangular envelope.

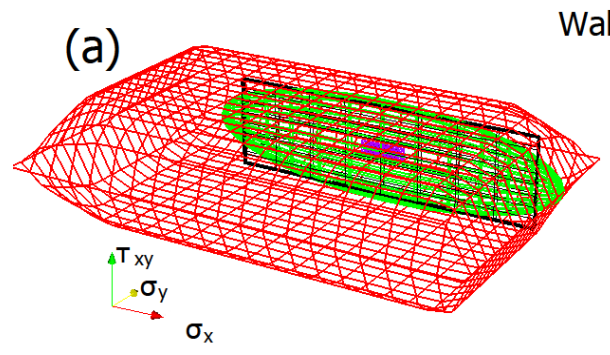

Wall1 Element 378

(b)

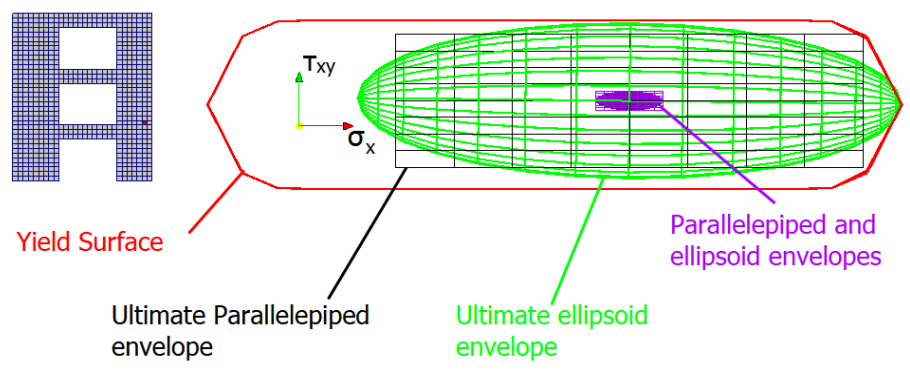

Figure 9: Yield surface, parallelepiped, ellipsoid envelopes and ultimate parallelepiped and ellipsoid envelopes - perspective view (a) and section view (b).

It is however important to note that, after performing analysis, the safety margins are always greater than 1 , and consequently design is safe.

\section{CONCLUSIONS}

This paper presented an efficient assessment strategy for performing capacity checks of shell elements subjected to seismic multicomponent excitations. The proposed method is based on the use of the well-known three-layer model of Marti, in order to reduce the dimension of the problem. The algorithms developed in this work facilitate the comparison of the response-spectrum-based envelopes to prescribed capacity surfaces for the purpose of assessing the seismic safety margin of reinforced concrete walls and slabs for nuclear power 
plant structures. The results of this algorithm are the available seismic safety margin on an element-byelement basis over the entire structural element area. The algorithms are suitable for evaluation the seismic margin of structures, whose behaviour remains essentially elastic for forced-based limit states and cannot be used for limit states outside the elastic response range of the structure.

The proposed numerical strategy was applied to a 3D structure made of reinforced concrete walls in order to find the seismic safety margin. As expected, the obtained results highlighted once again, the intrinsic conservatism implied by the verification methods commonly used in practice. Use of elliptical envelope method predicted systematically higher safety margins than those given by the application of the rectangular envelope method. As an example, and within a certain region of the structure, the safety margin given by the elliptical envelope method was as high as 1.3 times greater than that given by the rectangle method. It should however be noted, that this study was concerned with a particular case. More systematic and parametric studies considering a wider variety of geometric configurations for wall systems could lead to different results, possibly demonstrating more differences between the elliptical envelope and the classical rectangular method. Finally, under the assumption of identical horizontal motion intensities, it is clear that using the rectangular envelope method for assessment, although conservative, is inaccurate. Obviously, the most reliable way of calculating seismic safety margins should be based on the elliptical envelope strategy.

\section{REFERENCES}

[1] RCC-CW. (2014). "Rules for Design and Construction of PWR Nuclear Civil Works", Draft, AFCEN.

[2] Capra, A., Maury, J-F. (1978). "Calcul automatique du ferraillage optimal des plaques ou coques en béton armé". Annales de l'Institut Technique du Bâtiment et des Travaux Publics, No. 367 (in french).

[3] Cast3M-CEA. http://www-cast3m.cea.fr/

[4] CEN. (2004). European standard EN 1998-1, Eurocode 8: "Design of structures for earthquake resistance, Part 1: General rules, seismic actions and rules for buildings". Commité Européen de Normalisation, Brusells, (December).

[5] CEN. (2004). European standard EN 1992-1-1, Eurocode 2: "Design of concrete structures, Part 1-1: General rules, and rules for buildings". Commité Européen de Normalisation, Brusells, (December).

[6] EPRI. (1994). "Methodology for Developing Seismic Fragilities", prepared by J.R. Benjamin and Associates, Ink Mountain View California and RPK Structural Mechanics Consulting Yorba Linda California, TR-103959, Project2722-23, Final Report (June).

[7] Gupta O, Lacoste AC. (2006). "Prise en compte du risque sismique à la conception des ouvrages de génie civil d'installations nucléaire de base à l'exception des stockages à long terme des déchets radioactifs". Guide de l'Autorité de Sûreté Nucléaire. ASN/GUIDE/2/01. Autorité de Sûreté Nucléaire.

[8] Der Kiureghian, A. (1979). "On Reponses of Structures to Stationary Excitation". Report No. UCB/EERC79/32. Earthquake Engineering Research Centre, University of California, Berkeley.

[9] Jaeger, T. (2014). "Extended sandwich model for reinforced concrete slabs: Shear strength with transverse reinforcement". Engineering Structures 74 218-228.

[10] Lourenço, P.B., Figueiras, J.A. (1995). "A solution for the design of reinforced concrete plates and vaults”. J. Struc. Engrg., ASCE, 121(5), 815-823.

[11] Marti, P. (1990). "Design of Concrete Slabs for Transverse Shear", ACI Structural Journal, V.87, No.2 (March-April), 180-190.

[12] Mayboom, J. (2002). "Limit Analysis of Reinforced Concrete Slabs". Institute of Structural Engineering Swiss Federal Institute of Technology Zurich.

[13] Menun, C., Der Kiureghian A. (2000). "Envelopes for Seismic Response Vectors. I: Theory". Journal of Structural Engineering 126 (April), 467-473.

[14] Menun, C., Der Kiureghian, A. (2000). "Envelopes for seismic response vectors: II. Application". $J$. Struct. Eng. 126 (April), 474-481.

[15] Menun, C. (2004). "Strategies for identifying critical seismic response combinations". Earthq. Spectra 20

[16] Palacio, K. (2007). "Advances on the Design of Thin Surface Structures in Reinforced Concrete", PhD Thesis, Civil Engineering Department at the University of Minho, Portugal, July 2007, http://hdl.handle.net/1822/7078.

[17] SMART. (2013). http://www.smart2013.eu/ 